\title{
The use of emerging technologies for authentic learning: A South African study in Higher Education
}

Authors

Vivienne Bozalek

Daniela Gachago

Lucy Alexander

Kathy Watters

Denise Wood

Eunice Ivala

Jan Herrington

\begin{abstract}
It is now widely accepted that the transmission of disciplinary knowledge is insufficient to prepare students leaving higher education for the workplace. Authentic learning has been suggested as a way to bring the necessary complexity into learning to deal with challenges in professional practice after graduation. This study investigates how South African higher educators have used emerging technologies to achieve the characteristics of authentic learning. A survey was administered to a population of 265 higher educators in South Africa who self-identified as engaging with emerging technologies. From this survey, a sample of twenty one respondents were selected to further investigate their practice through in-depth interviewing using Herrington, Reeves \& Oliver's (2010) nine characteristics of authentic learning as a framework. Interrater analysis undertaken by five members of the research team revealed both consistencies and differences among the twenty one cases across the nine elements of authentic learning. The highest levels of authenticity were found for the elements authentic context and task, and the lowest for articulation. Furthermore, there was a moderate correlation identified between levels of authenticity and the role played by emerging technologies in achieving the authenticity, showing a potentially symbiotic relationship between them.
\end{abstract}

\section{Introduction}

Despite efforts by higher education institutions (HEIs) to address the disjuncture between curriculum design and what is required of working professionals, students internationally and more particularly in South Africa, still graduate unprepared to confront the realities of the twenty first century workplace (Herrington, Mantei, Herrington, Olney, \& Ferry, 2008; Lombardi, 2007). Herrington et al. (2010) argue that higher education faces the challenge of a curriculum that remains largely decontextualised. Consequently, they call for incorporation of what they propose as the nine elements of authentic learning in this sector, in order to enable more contextually relevant learning. Using this model, we argue that emerging technologies, which Veletsianos (2010, p. 17) defines as the 'tools, technologies, innovations, and 
advancements utilized in diverse educational settings to serve varied education-related purposes', have the potential to support authentic learning. This definition and conceptualisation of emerging technologies regards context to be very important -what is regarded as emerging in one context may not be in another and vice versa. In South Africa, with the exception of Amory's (2012) work, there is currently limited research regarding these intersections. This paper aims to contribute evidence for the potentially symbiotic relationship between emerging technologies and authentic learning. It reports on a research project conducted with selected South African higher educators to explore their teaching practices for evidence of what Herrington et al. (2010) term the elements of authentic learning. Simultaneously, we investigated the contribution of emerging technologies to achieving this authenticity, by which we mean the level of authentic learning across Herrington et al.'s (2010) nine elements.

\section{Literature Review}

Over the past three decades, increasing international attention has been focused on the effectiveness of higher education in addressing graduate readiness for the workplace by developing relevant competences, capabilities and attributes through appropriate teaching practices (Billett, 2009; Department of Education, 2007). In part, this has been a reaction to the focus on imparting disciplinary knowledge in higher education, which has tended to decontextualize knowledge, leaving graduates unprepared to apply this knowledge in different contexts when the need arises (Brown, Collins, \& Duguid, 1989; Herrington et al., 2010; Lave \& Wenger, 1991; Schön, 1983).

As early as the late 1990s, a set of conditions termed authentic learning was coined by Herrington and Oliver (2000). They argued that the adoption of authentic learning as a pedagogical model can help to better prepare students for professional practice. To this end, Herrington et al. (2010, p. 18) identified nine elements, which they argue characterise authentic learning designs. They are:

1. Authentic context - for Herrington et al. (2010), the curriculum should not be simplified and broken up into step-by-step processes, but should rather mirror real world situations in order to reflect the way knowledge would be used in these situations. An authentic context leads to an intense feeling of engagement with the learning, where the learner experiences what Herrington et al. (2010, p. 92) refer to as the 'willing suspension of disbelief'.

2. Authentic task- the task should be ill-defined, complex, comprehensive, and completed over an extended period of time, mirroring activities that are relevant to the kinds of problems to which knowledge is applied in the real world. 
3. Access to expert thinking and modeling of processes - both students themselves who are more knowledgeable others (Vygotsky, 1978) and lecturers can enhance learning for others through sharing their different levels of expertise.

4. Provide multiple roles and perspectives - students should be enabled to examine problems from more than one point of view to ensure expertise in areas.

5. Support collaborative construction of knowledge - providing opportunities for students to work on a common task which is assessed collaboratively is important.

6. Promote reflection to enable abstractions to be formed - it is essential to provide opportunities for students to reflect in action, while completing their tasks and on action, after tasks have been completed (Schön, 1983). Reflection is seen in authentic learning as a social and interdependent, working in collaborative groups, rather than individual process. In this way, students should be able to compare their ideas to more knowledgeable others, associating and integrating new knowledge into their conceptual frameworks.

7. Promote articulation to enable tacit knowledge to be made explicit - opportunities should be provided for students to speak and write about their growing understanding within communities of professional practice and in public fora.

8. Provide coaching and scaffolding by the teacher at critical times - the idea is that rather than transmitting knowledge, the teacher's role is a supportive one, guiding and supporting students.

9. Provide for authentic assessment of learning within the tasks - the assessed task is the polished product at the end of the learning period which has taken a significant period to produce and is integrated with the learning tasks.

The conceptual foundations of authentic learning are linked to Brown et al.'s (1989) theory of 'Situated Cognition', which they based on the study of highly successful learning interactions. Key to the achievement of such learning interactions is the provision of apprenticeship-type learning in situations to '... reflect the way the knowledge will be useful in real life' (Collins, 1988, in Herrington et al., 2010, p.14). Also important to their conception of learning for real world application is an affirmation of the social embeddedness of practice. 


\section{Emerging Technologies and Authentic Learning}

In Herrington et al.'s (2010) survey of the literature on the contribution of emerging technologies to enhance learning and Lombardi's (2007) Educause paper, it is argued that emerging technologies are able to promote a number of the characteristics of authentic learning, such as collaboration across distance, sharing of results, communicating with experts and access to online research communities. Emerging technologies that seem to offer opportunities for authentic learning are those which promote collaboration (e.g. digital databases, social networking tools and referencing tools), enable joint construction of knowledge (e.g. role-plays, problem-based activities, case-based learning, discussion forums and virtual communities of practice), and allow for individual or group articulation (e.g. blogs, e-portfolios, and video-capture devices) (Herrington et al., 2010; Lombardi, 2007). In particular, Herrington et al.'s (2010) most recent research on mobile technologies, such as the use of podcasts and digital stories for authentic learning, is of interest to this study. The ubiquity of mobile technology in South Africa holds promise for a wider adoption of technology in teaching and learning (Donner, 2008). For example, a recently published South African example on the use of technologies for promoting authentic learning (Amory, 2012) describes developing an authentic intervention in postgraduate teacher education. In this intervention, mediated learning experiences were provided which transformed students' worldviews and assisted them to engage with educational technologies as tools in order to construct collective knowledge.

\section{Methodology}

The two research questions guiding this study were:

- Is there evidence that South African higher educators who were interviewed for this study were incorporating any of the nine elements of authentic learning as defined by Herrington et al. (2010)?

- To what extent have emerging technologies contributed to achieving the nine elements of authentic learning in these case studies?

The reported findings in this paper form part of a larger study, conducted in 2011, which surveyed the use of emerging technologies amongst 262 South African higher educators (for further information on this study (see Bozalek, Ng'ambi, \& Gachago, in press and the Emerging ICTs in Higher Education blog cf. http://www.emergingicts.blogspot.com). From the open ended responses to the survey, 75 educators whose practices showed evidence of authentic learning were identified by one research team member, who is a co-author on this paper. The researchers as a group then reviewed these survey responses and narrowed the identified 75 educators to 28 educators whose practice showed evidence of one or more dimensions of authentic learning. These educators were invited to participate in the next stage of this research: 
21 of the 28 accepted. Prior to commencement of the study, ethical clearance was obtained by the principal investigator, participants gave informed consent and respondents were anonymised.

Data were collected by five researchers, three of whom are co-authors on this paper, through semi-structured face-to-face, telephonic or online interviews from April to May in 2012. As shown in Table 1, male and female respondents were evenly distributed, and ranged in teaching experience from one to more than 20 years. Most of the respondents in the sample were from the Applied Sciences, while Natural Sciences, Education, Humanities and Commerce were also represented in small numbers. Educators came from seven differently placed HEIs in terms of geographical location, demographics and resource allocation (Bozalek \& Boughey, 2012).

Table 1: Demographics - Higher Educator Cases

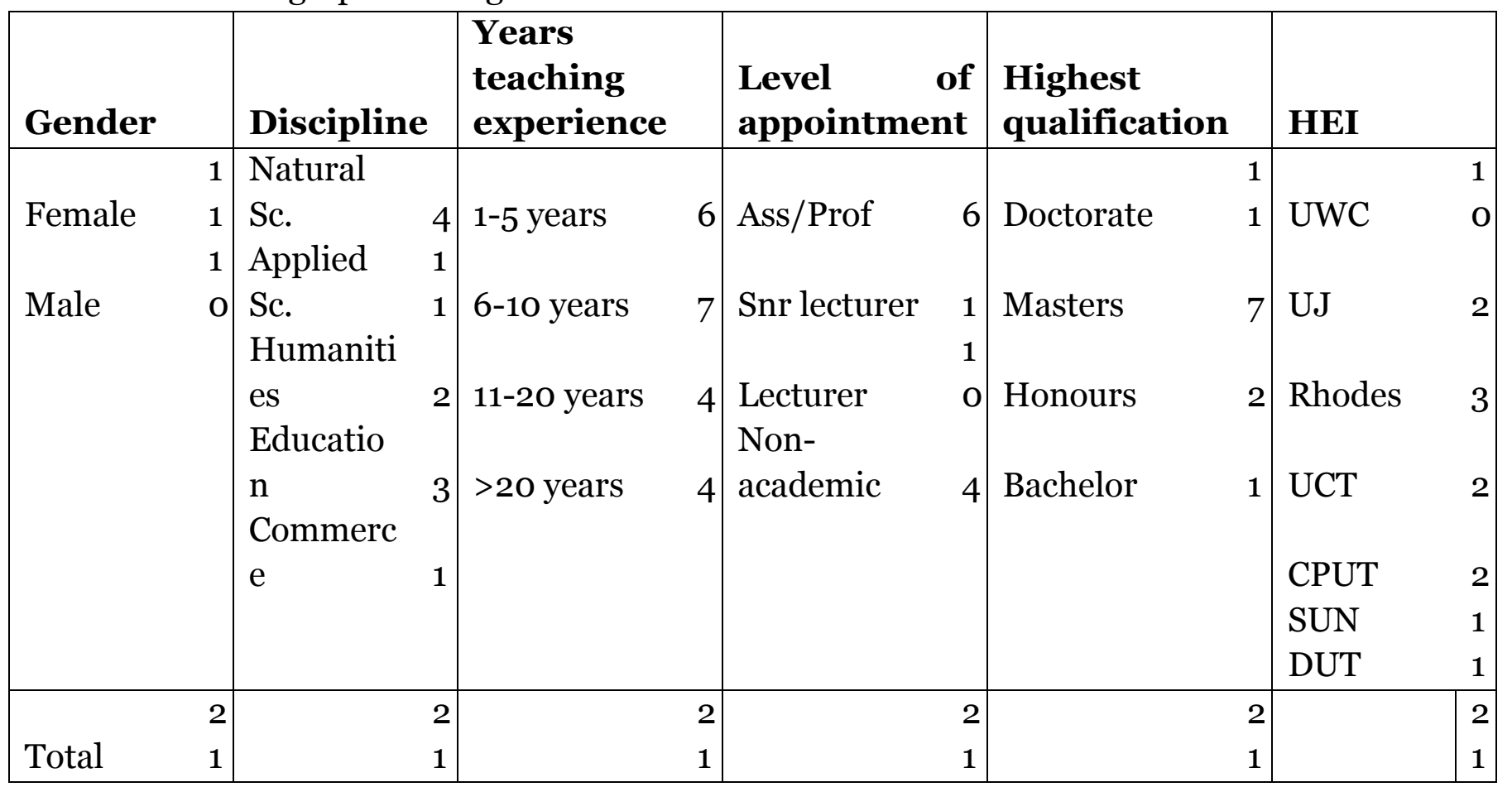

Interviews were structured along the nine elements of authentic learning using questions suggested by Herrington in her online videos (c.f. Herrington, 2011). From these interviews, cases were developed. In a similar fashion to Amory's (2012) study, where students used a Likert scale to assess the course along authentic learning elements, the cases in this study were analysed by five members of the research team, four of whom are co-authors of this paper, to establish the extent to which each of Herrington et al.'s (2010) elements were achieved by each educator. Using a scale ranging from $\mathrm{O}$ to 2, scores were assigned depending on the strength of evidence of authentic learning, with o signifying 'no evidence'; 1 indicating 'weak evidence'; and 2 
signifying 'strong evidence'. This data was captured into MS Excel and member checks were performed to verify the scores.

Two sets of scores were calculated:

1 composite scores and percentages of authenticity per case to establish each case's level of authenticity (composite score across all authentic elements per case, max score: 90)

2 composite scores and percentages for each authentic learning element (composite score of each element across all 21 cases, max score: 210)

In relation to Research Question 2, five members of the team analysed the 21 cases in terms of the contribution of the educator's use of emerging technologies to each specific authentic learning element (again scored using a scale ranging from o to 2). A statistical analysis and graphics software package NCSS 8 (see http://www.ncss.com) was used to create a scatterplot, establishing the correlation between the use of emerging technologies and the level of authentic learning of each individual case.

To test for interrater reliability among the five researchers, Free-Marginal Multirater Kappa was calculated, which is used in fields of content analysis and meta-analysis when a researcher wants to determine how well raters agree on the coding of nominal variables, in particular for cases, in which raters' distributions of cases into categories are not restricted (Randolph, 2005). This is the case when a rater is free to assign cases to categories with no limits on how many cases must go into each category (as opposed to e.g. Fleiss' Multirater Kappa, see Brennan \& Prediger, 1981).

\section{Findings}

Research question 1: Evidence of authentic learning across 21 case studies

To measure whether participating South African higher educators' teaching practices showed evidence of authenticity, five members of the research team rated the 21 cases along the nine elements of authentic learning using a scale of $0-2$. The maximum composite score per case for authentic learning was 90, with 10 points per element. A composite score of all cases per element was also calculated, totalling a maximum of 210 points. Results of this analysis are presented per element in Figure 1. The composite scores were converted to percentages for ease of comparison. 
Figure 1: Level of authenticity per authentic learning element

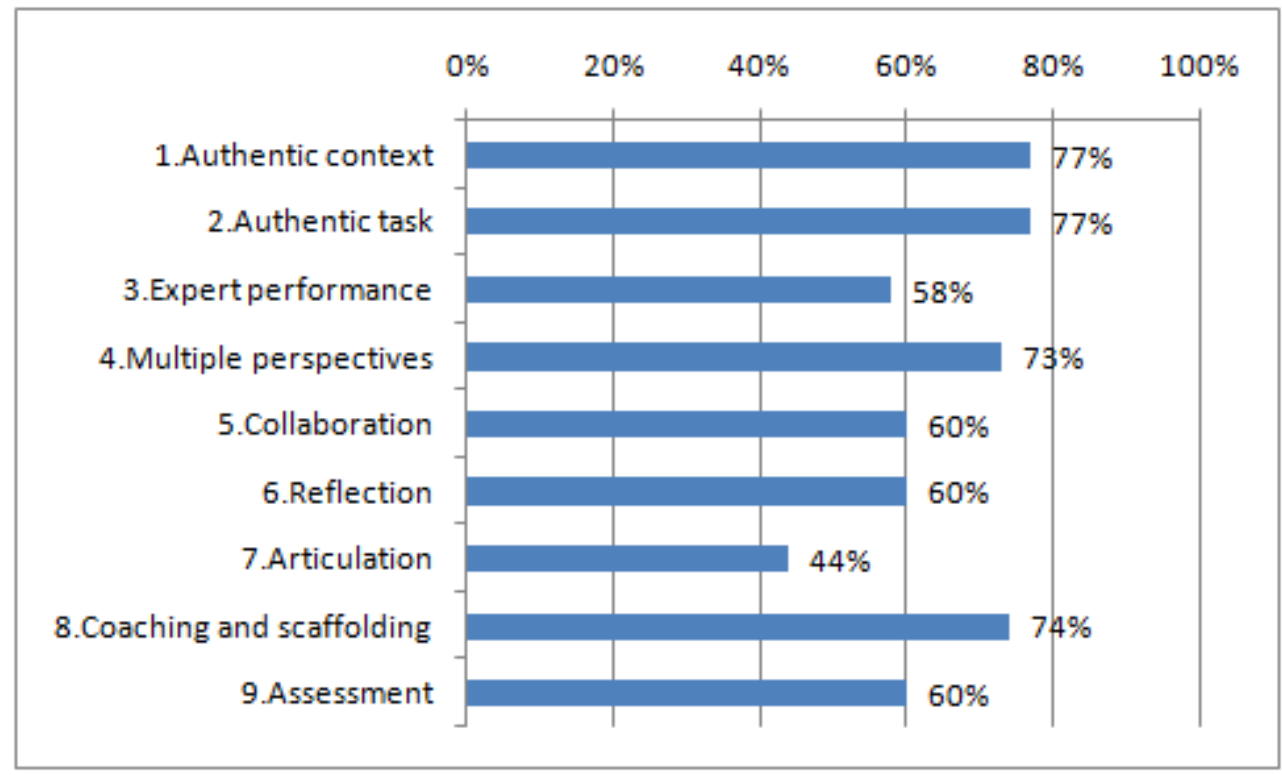

The composite authenticity scores were highest for four of the elements: most prominent was the use of authentic tasks and authentic contexts (in $77 \%$ of the cases respectively); coaching and scaffolding was also evident in $74 \%$ of the cases, while the incorporation of multiple perspectives was visible in $73 \%$ of the cases. Authentic tasks and authentic contexts scored highest possibly because these elements most literally recreate aspects of the activities and real world contexts of their professions. This was evident in cases such as Physiotherapy, Community Health and Teacher Education. This was followed by coaching and the incorporation of multiple perspectives, which require a higher degree of educator sensitivity to learner or contextual needs. Assessment, collaboration, reflection and expert performance all scored around 60\%. The lowest scored element was articulation (44\%).

Research question 2: Contribution of emerging technologies to the achievement of authentic learning elements

Our second research question examined the extent to which emerging technologies contributed to the achievement of the nine elements of authentic learning in the 21 cases.

Figure 2 shows the contribution of emerging technologies in the attainment of the various dimensions of authentic learning across the 21 cases. Findings show that emerging technologies made the most impact on the attainment of authentic context and authentic task, followed by coaching and scaffolding, and multiple perspectives. The least impact can again be seen in achieving articulation. 
Figure 2: Contribution of emerging technologies to the achievement of authentic learning

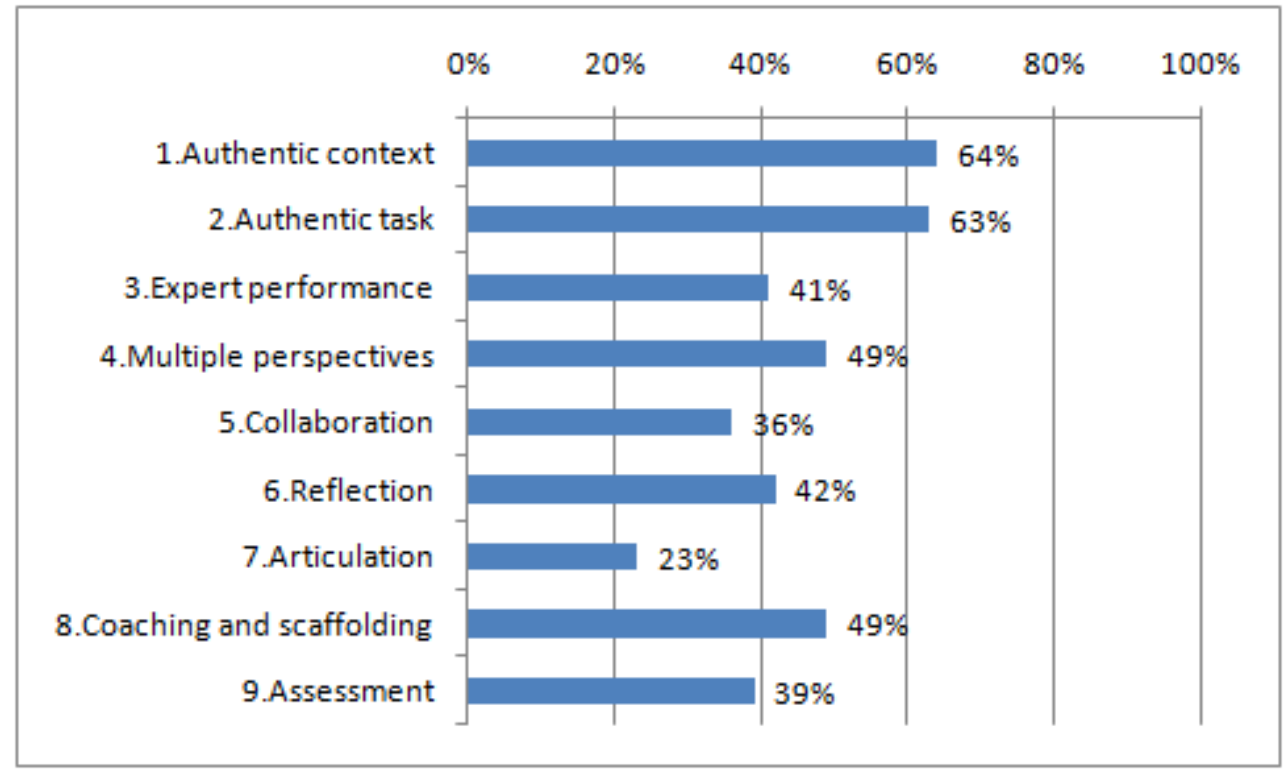

The scatterplot (Figure 3) on the use of emerging technologies and the level of authentic learning per individual case study shows a strong correlation $(\mathrm{r}=0.79, \mathrm{p}<0.001)$ between the total authenticity score across all elements and the emerging technologies score (see Figure 3 below). This indicates that the emerging technologies score was a good predictor for the authentic learning score and vice versa. This finding suggests a symbiotic as opposed to a simple cause-effect relationship between emerging technologies and authentic learning 
Figure 3: Correlation between the use of emerging technologies and the level of authentic learning per individual case study (highest scoring cases 1-5 labelled

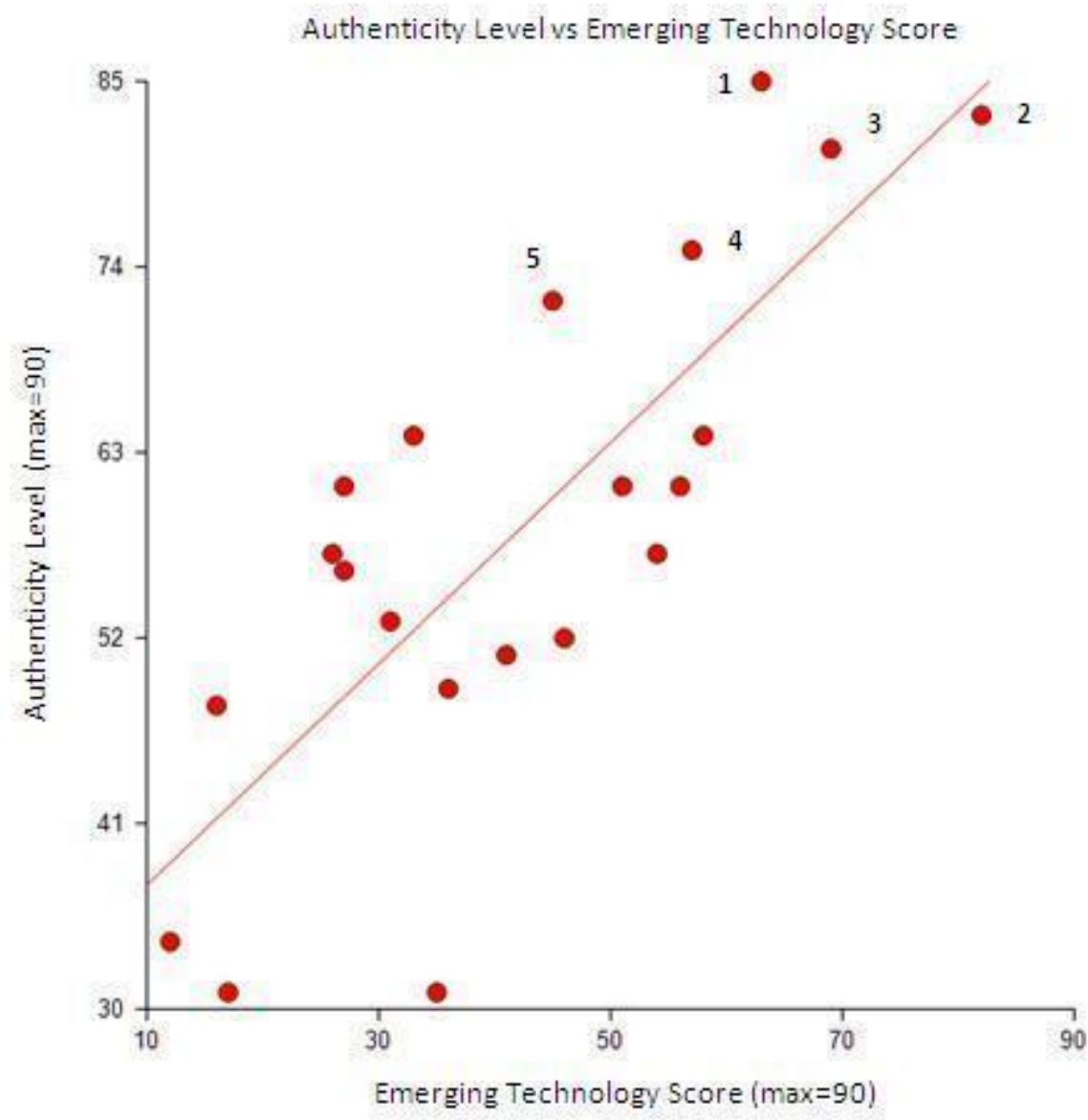

This symbiosis between authentic learning elements and emerging technologies was most evident in the following five of the twenty one case studies, which scored the highest in terms of authenticity and emerging technologies (see Figure 3: case studies 15 are indicated):

1 In a citizen journalism project in Media Studies, students worked with high school learners from disadvantaged communities, to collaboratively produce digital media videos on social issues using mobile phones. These were then published in a local newspaper and on YouTube. Elements of authentic learning such as collaboration, public articulation of authentic product, real world relevance were thus supported through the use of digital video, an emerging technology.

2 In a five country consortium of academics an online course on women's health and well-being was designed and implemented, where students across five countries 
worked collaboratively across their respective contexts and produced a final research project using wikis. This emerging technology allowed a highly contextualised and distributed collaborative project, allowing access to various expert networks. Intensive online tutoring and support accompanied the process.

3 In a teacher education project, final year students reflected on their journeys to becoming teachers through the medium of digital stories. The multimodality allowed for complex meaning-making and the development of multiliteracies which were carefully scaffolded by a team of interdisciplinary experts.

4 In a course located in Biodiversity studies, students reflected through individual blogs on adaptive water management of their own fish, which they maintained in a fishbowl throughout the course. This allowed for the lecturer to introduce complex scientific concepts by linking them to students' authentic experiences.

5 In a physiotherapy course, students were taught to engage in critical reasoning through active dialogues in the classroom and through publishing their critical reflections through various channels, such as wikis and Google docs. These technologies allowed not only students and facilitators, but the public to engage in a conversation about critical reasoning

\section{Interrater reliability}

With regard to the reliability of the findings, results revealed an average Free-Marginal Multirater Kappa correlation factor of 0.43, which Landis and Koch (1977) categorise as moderate agreement for the general evaluation across the evidence of the nine elements of authentic learning and 0.27 (fair agreement) for the evaluation of the contribution of emerging technologies for each of the nine elements. Where high levels of divergence were evident, the researchers discussed their scoring rationale and scores were adjusted accordingly.

\section{Discussion and conclusion}

The study was aimed at exploring how South African higher educators are using emerging technologies to achieve authentic learning.

The findings of the study indicate that respondents applied the nine elements of authentic learning to various degrees in their teaching practices. The elements of authentic context and authentic task were the most commonly used, with articulation being the least evident, confirming findings from previous studies (Amory, 2012). The latter may be partly due to the way in which assessment is mandated in most HEIs, which is often focused on traditional exams rather than inquiry-based learning and authentic assessment potentially mitigating against more creative and authentic ways of students' presenting and publishing their work. A similar argument might be made for the low score on authentic assessment, which may also to some extent be attributed to constraints in the institutions assessment policy. 
It was also clear from the analysis that the alignment of the authentic learning principles with each learning environment was not a case of absolutes. The results confirmed that while useful as a checklist in the design phases of a pedagogical approach, the elements are better viewed as continuums in evaluating how well they align with a particular course intervention

(cf. http://authenticlearning.info/AuthenticLearning/Evaluation.html). The data confirmed that an intervention can be weak on one or two elements while still presenting a substantially authentic experience for students because of the strength of the remaining elements.

The process adopted for analysis of the data by five researchers in this project provided interesting insights into the role that context plays in the identification of technologies that can be regarded as 'emerging' in a given situation. Although the rating process followed a quantitative data analysis method, it became apparent in discussions around divergence of ratings and views that individual understandings of the concept of emerging technologies varied widely based on the individual researcher's context. This was confirmed by the variance evident between raters when examining Free-Marginal Multirater Kappa. While as noted in the findings section, Free-Marginal Multirater Kappa demonstrated a moderate agreement overall, examination of correlations between individual raters revealed a slightly lower level of consistency for items relating to the role of emerging technologies in supporting authentic learning. This finding can be explained by the varied disciplinary backgrounds and geographical contexts of the raters, and reflects the role of context as foregrounded in the definition of emerging technologies put forward by Veletsianos (2010, pp. 18-19). We see the diverse experiences of an international team as an enrichment rather than a limitation. By involving five researchers, we aimed to improve the robustness of the research design.

In summary, this study shows that educators scoring highly on the use of emerging technologies to facilitate authentic learning are embedded in a larger communities of practice, drawing from a cross pollination of ideas, foregrounding the importance of sharing and collaborating in a field that is constantly changing. The results also show that there is a symbiotic, rather than a simple cause-effect relationship between emerging technologies and transformative teaching and learning approaches like authentic learning. While this study has focused strongly on teachers' pedagogical practices, more research is needed to shed light on particular conditions and other important factors-such as the educator's teaching, and learning assumptions and beliefs-that impact on this relationship. 
Implications for practice derive principally from the combination of emerging technologies and a strong theoretical framework in authentic learning, specifically the nine guiding principles that are easily accessible for educators and practitioners to design, implement and evaluate their technology-based learning environments. Moreover, for a wider community to adopt similar practices, it would seem necessary to develop and implement professional learning and staff development initiatives on the interplay of emerging technologies and authentic learning, for induction and scaffolding of pedagogical practice. Such programs could assist educators to review their curricula to include complex and engaging tasks, illustrated well in this study in the exemplars of successful cases. While some elements of authentic learning, such as reflection and collaboration, can readily be instantiated in courses by individuals and teams of committed teachers, it could take a change in institutional culture and policy to facilitate other elements, such as authentic assessment. Removing institutional impediments would enable educators to focus more fully on the synergies and affordances offered through the pairing of strong theory and powerful technologies.

\section{Acknowledgements}

The authors acknowledge with gratitude funding provided by the National Research Fund (NRF) which made the research project reported on in this paper possible. The views of the authors expressed in this paper do not necessarily reflect those of the NRF. The authors also wish to acknowledge the other team members in the NRF project who have contributed to the intellectual and practical development of the emerging technologies in higher education project - specifically Dianne Dawes and Roshini Pillay. Particular appreciation is extended to the lecturers who gave up their time to be interviewed. 


\section{Bibliography}

Amory, A. (2012). Tool-mediated authentic learning in an educational technology course: a designed-based innovation. Interactive Learning Environments, (October), 117.

Billett, S. (2009). Realising the educational worth of integrating work experiences in Higher Education. Studies in Higher Education, 34(7), 827-843.

Bozalek, V., \& Boughey, C. (2012). (Mis)Framing higher education in South Africa. Social Policy and Administration, 46(6), 688-703.

Bozalek, V., Ng'ambi, D., \& Gachago, D. (in press). Transforming teaching with emerging technologies: Implications for Higher Education Institutions. South African Journal of Higher Education.

Brennan, R. L., \& Prediger, D. J. (1981). Coefficient kappa: Some uses, misuses, and alternatives. Educational and Psychological Measurement, 41, 687-699.

Brown, J. S., Collins, A., \& Duguid, P. (1989). Situated cognition and the culture of learning. Educational Researcher, 18(1), 32-42.

Department of Education. (2007). The Higher Education Qualifications Framework. Pretoria: Government Gazette.

Donner, J. (2008). Research Approaches to Mobile Use in the Developing World: A Review of the Literature. Information Society, 24(3), 140-159.

Herrington, J. (2011). Authentic Learning. Retrieved February 13, 2013 from http://authenticlearning.info/AuthenticLearning/

Herrington, J., Mantei, J., Herrington, A., Olney, I., \& Ferry, B. (2008). New technologies, new pedagogies: Mobile technologies and new ways of teaching and learning. Ascilite20o8 Melbourne (pp. 419-427).

Herrington, J., \& Oliver, R. (2000). An instructional design framework for authentic learning environments. Educational Technology Research and Development. Educational Technology Research and Development, 48(3), 28-48. Retrieved February 13, 2013 from http://researchrepository.murdoch.edu.au/5251

Herrington, J., Reeves, T. C., \& Oliver, R. (2010). A guide to authentic e-learning. New York: Routledge. 
Landis, J. R., \& Koch, G. G. (1977). The Measurement of Observer Agreement for Categorical Data Data for Categorical of Observer Agreement The Measurement. Biometrics, 33(1), 159-174.

Lave, J., \& Wenger, E. (1991). Situated learning: Legitimate peripheral participation. Cambridge: Cambridge University Press.

Lombardi, M. M. (2007). Authentic Learning for the 21st Century: An Overview (ELI paper 1: 2007). (D. G. Oblinger, Ed.) EducauseLearningInitiative. Retrieved February 13, 2013 from http://net.educause.edu/ir/library/pdf/ELI3009.pdf

Randolph, J. J. (2005). Free-Marginal Multirater Kappa (multirater kfree): An Alternative to Fleiss' Fixed- Marginal Multirater Kappa. University of Joensuu Learning and Instruction Symposium. Joensuu, Finland.

Schön, D. A. (1983). The reflective practitioner: How professionals think in action. New York, USA: Basic Books.

Veletsianos, G. (2010). Emerging Technologies in Distance Education. Theory and Practice. Edmonton: AU Press. Retrieved February 13, 2013 from http://www.aupress.ca/books/120177/ebook/99Z_Veletsianos_2010-

Emerging Technologies in Distance Education.pdf

Vygotsky, L. (1978). Mind in society. The Development of Higher Psychological Processes. Cambridge: Harvard University Press. 